\title{
A Survey on Thermal Aware Routing Protocols in Wireless Body Area Network
}

\author{
Hagar Mahmoud ${ }^{1}$, Etimad Fadel ${ }^{1}$ \\ Department of Computer Science, Faculty of Computing and Information Technology, King Abdulaziz University, \\ Jeddah, Saudi Arabia1 ${ }^{1,1}$
}

\begin{abstract}
Wireless Body Area Network (WBAN) is a wireless network of wearable devices in or around the human body to serve a variety of applications. In healthcare, sensors might be implanted in or around the human body to monitor and detect abnormal behaviors. In addition to the resource limitations for WBAN, more challenges are existing when applying WBAN for human body applications as in the medical field. Coming to the safety aspect, a major consideration needed to address the heating effects due to wireless communication which cause damage to the surrounding tissues. Therefore, thermal aware routing protocols were proposed by researchers to consider the temperature of the implanted medical devices while routing. This survey presents a state of are for thermal aware routing protocols as a step for coming research addressing the same issue. It provides a comparison between the existing protocols, to finally list the routing design challenges for future research.
\end{abstract}

Keywords: WBAN, thermal-aware, routing protocol, hotspot, temperature.

\section{INTRODUCTION}

Wireless Body Area Network (WBAN) is a network of wearable devices in or around the human body which uses wireless communication to serve a variety of applications. The applications for using WBAN include remote health/fitness monitoring, military and sports training, interactive gaming, personal information sharing, or secure authentication. In healthcare, WBAN enables assisting the independent living, monitoring patient, early detection for diagnosis, and provide medical prescription remotely [1]. In WBAN, the wireless sensor devices consist of a radio transceiver, processor, and battery source [2]. It aims to collect the information (e.g. data about the patient) and send it to the sink node. Reaching the sink node can be done directly or indirectly via relay nodes. Then, the sink node is responsible for sending the sensed information to its corresponding healthcare center.

WBAN is a subset of WSN (Wireless Sensor Network) with more environmental restrictions and limitation of resources. The human body as an environment is significantly smaller than the usual WSN environments and requires a different type and frequency of monitoring. Despite the existing routing protocols for WSNs, these protocols cannot be used for WBANs due to its cruel requirements. For example, minimizing routing overhead and maximizing throughput is the main consideration in WSN than reducing energy consumption as in WBAN.

The reliability and efficiency of WBAN depend on how fast, and accurate the process for sending and responding to the events and share data between the nodes; the selected routing protocol is the key factor on this. In addition to the resource limitation, the safety aspect is the major concern for designing WBAN routing inside the human body. In fact, the heat dissipation from devices causes tissue damage. On the other hand, changes in body posture make frequent wireless links breakage due to topology changes.

In this paper, a list of thermal aware routing protocols for WBAN is introduced and a comparison is done between them according to routing algorithm focus. The currently available surveys are dealing with performance evaluation for the protocols. The rest of this paper is organized as follow: Section II provides background about WBAN, its architecture, it's standard, and challenges exist especially in medical filed. In section III, a survey for thermal-aware routing protocols is provided. Then, in section IV the tools and appropriate application for each routing is provided. After that, section $\mathrm{V}$ provides the routing design challenges to conclude the work in section VI.

\section{A. WBAN Architecture}

\section{WIRELESS BODY AREA NETWORK}

The architecture for having a WBAN composed of three communication tiers [2]. First, tier-1 as Intra-BAN. it is the one located internally inside the body. The communication within this tier occurs between sensors inside the body and between intrabody sensors with the controller to send the data outside. Second, tier-2 as Inter-BAN. it is the middle central controlling layer consists of an intelligent electronic device. It acts as a broker to gather the information from intrabody sensors and pass it to the workstation (tier-3) via wireless communication. Third, tier-3 as Extra-BAN which 


\title{
International Journal of Advanced Research in Computer and Communication Engineering
}

\author{
Vol. 8, Issue 9, September 2019
}

analyzing the received data in order to provide the appropriate response for the monitored abnormal behavior; it contains the DB.

Building WBAN requires special consideration on the hardware and network level. Three main hardware requirements needed for having WBAN which are biosensor [3] for detecting signs inside the human body, gateway acting as a controller for biosensors to collect the information and store it in local memory, and monitoring server which consists of a database for storing the further needed processing. The network requirements for BAN are communication range ( 2 to 5 meter), interference avoidance, network density (2-4 networks per m2), maximum of 256 devices per network, security to ensure integrity of data, latency to max of 125 for medical field and enable for prioritization of packages to support the medical urgent needs[1].

\section{B. IEEE 802.15.6}

The currently available Wireless Local Area Networks (WLAN) /Wireless Personal Area Network (WPAN) technologies, for example, Bluetooth, Zigbee and Wi-Fi (under IEEE 802.11 standard) are not proper for WBANs [4] since they do not comply with the medical and important correspondence guidelines for some application environments. For that, the IEEE 802.15.6 standard was presented to fulfill the requirements of a monitoring system which operates on, in or around the human body.

IEEE 802.15.6 is a standard for short-range, low power, and highly reliable wireless communication in, on and around the human body. telemedicine, and assisted living are examples for the applications supported by this standard. The standard is satisfying most of the medical application requirements in terms of throughput. However, it is not satisfying the application required high-quality video and audio transmission[1].

\section{Challenges for WBAN in Medical Field}

In addition to the restrictions for WBAN devices, humans' body is a challenging environment which highly affects the communication between nodes in such network [5]. WBAN device characteristics are low power, limited computational tasks, limited communication ranges and needs for robustness to ensure increase lifetime for devices as replacement is not easy. The challenges related to the human body are: First, movement of limbs which effects on the network such as shadowing and change in a network topology. Second, the different size and heterogeneous nature of organs. Third, the heating effects on terahertz frequency and its damage to human tissue are one of the major safety issues

\section{III.THERMAL-AWARE ROUTING PROTOCOLS}

Increasing device heat can affect surrounding tissues and cause damage if it exceeds a certain level. The sources for heat generation in routing are power squandering by circuits of sensor node and radiation from the antenna [6]. There are two important measures exist to calculate the heat increase [6, 7]. First, the Specific Absorption Rate (SAR) to measures the absorption rate for human tissue when exposed to a Radio Frequency (RF) electromagnetic field. Second, Penne's Bioheat Equation Method (PBHTE) to determine the rate of rising in temperature. Finite-Difference TimeDomain Method (FDTD) [8] and sensor-centric thermal-rise model [9] are used for modelling the heating affects.

Thermal-based routing protocols is the type of routing which considers the temperature of the devices in routing. This type usually suffers from complexity and overhead. All thermal aware routings are tending to avoid transmission of the data via a hotspot. The hotspot is the node which is exceeding threshold temperature value. There are three types of routing used on WBAN protocols. First, proactive routing where each node has information about the neighbor nodes. Second, reactive routing where the node explores the information about the neighbors when there is a packet to be sent. Third, a hybrid which combines the benefits of two methods (e.g. TAER which use proactive in setup phase and reactive in data transmission phase). In our knowledge, we can also classify the routing types as per the techniques for handling hotspot nodes. First, the detection technique which detects that node is hotspot after passing through it, then take the decision to avoid it. Avoidance technique which expects that node will become a hotspot before passing to it.

Thermal-Aware Routing Algorithm (TARA) [6] is the first proactive routing protocol that is considering the temperature of the node in the routing process. It measures the temperature of the device based on a number of packets transmitted and received. The protocol was modeled using FDTD (Finite Difference Time Domain) method during the process of detecting the hotspot. A withdrawn packet technique is used if the next hop is a hotspot to spread the information about the hotspot nodes to other neighboring nodes. If the hotspot node is reached, the backoff algorithm is used to return the packet to find another alternative path. Packet delivery ratio is increased by this mechanism in comparison with shortest hop routing (SHR). After cooling down, this node can be selected again. The protocol is simple and has a significant impact on preventing the selection of hotspot nodes. However, it increases the number of retransmissions as the node will be back to search for alternatives after its already checked all the hotspot nodes [2]. 


\title{
International Journal of Advanced Research in Computer and Communication Engineering
}

\author{
Vol. 8, Issue 9, September 2019
}

Also, the time lag for alternative path searching is increased. Moreover, TARA is reducing the lifetime and reliability as it considers only heat for the decision. It also depends on single-hop communication only[10].

Least Temperature Routing (LTR) is proposed in [11] after TARA with some modification to enhance its retransmission issue. Three main differences exist in LTR. First, it measures the temperature according to the data communication focus. It forwards the packet to the coolest neighbor unless it exists on the list of recently visited nodes. In this case, the packet will be forwarded to the second coolest neighbor. Second, MAX_HOPS parameter exists for defining the threshold value of the number of hops so the bandwidth can't increase much. Third, LTR keeps track of the previously visited nodes to avoid an infinite loop. LTR shows a significant impact on reducing the retransmission rate supports but it increases the end-to-end delay as most of the nodes are involved in routing [2]. This also may increase the overall temperature of the network[5].

Adaptive Least Temperature Routing (ALTR) [11] appears as an advanced version of LTR. ALTR provide a new parameter MAX HOPs ADAPTIVE to reduce the delay appears on LTR. ALTR counts the number of hops and compares it with MAX HOPs ADAPTIVE. If the hop count is greater than MAX HOPs ADAPTIVE, it uses SHR (Shortest Hop Routing) and keeps using LTR otherwise. Also, ALTR uses proactive delay strategy. Based on this strategy, if the coolest neighbor temperature is greater than the current node temperature, the current node delays the packet by one unit of time before sending it to this coolest neighbor. This strategy saves energy for less connected topologies (e.g. ring) to reduce the temperature of nodes which will be used most of the time. This also safe the overall network temperature. However, it may waste network bandwidth through unnecessary transmissions. It also allows the packet traverse through hotspot [12].

The above three routing protocols are considering only the temperature for next-hop node; hence, they increase the number of hops and the total temperature of the entire network. Least Total Route Temperature (LTRT) protocol is proposed in [13] to consider the full route temperature. It converts the network into a weighted graph with temperature as weight. Then calculate the shortest route using Dijkstra algorithm to select the best route. Maintenance is done periodically to avoid the increase in the route temperature (the route which is short will be the highest temperature if kept in use.). The protocol shows a significant impact on reducing the average temperature rise in comparison with LTR and ALTR. It also reduces the number of hops, packet loss in comparison with LTR, ALTR and SHR. However, the overhead for the algorithm for calculating all paths and periodically update the information may affect the velocity of sharing the critical information especially in critical medical applications. It also (as TARA, LTR, ALTR) does not consider the data priority, residual energy of the node.

ATTEMPT [14] is a proactive thermal aware routing aims to reduce the energy consumption in comparison with multi-hop communication. It periodically senses the hotspot and breaks it's around links for a few rounds if the node reaches the threshold value. After a while, the link is re-established to be used on data transmission again. The protocol is handing two types of data. For emergency data, single-hop communication is used to directly send the data from node to sink to ensure no delay. For normal data, multi-hop communication is used to select the route with a minimum hop count. To find the best route, ATTEMPT considers a number of hops count from node to sink and the total energy of the route on the routing phase. Then TDMA is used in the scheduling phase for assigning a time slot for nodes to share its packets to sink. Finally, packets are sent by nodes to be processed and aggregated by the sink in the data transmission phase. The MATLAB simulation shows that the algorithm is showing an increase is the stability period and throughput in comparison with multi-hop communication.

M ATTEMPT [14] is similar to ATTEMPT with more stability and supports mobility by placing nodes with high data at a less mobile place (the higher the closer to sink). It arranges the nodes into levels as per the energy and data rate. It adds invitation phase to handle the link breakage for the network, a joint request will be sent by a child node to be under a parent. The parent will accept the node if its number of children less than 3 . Thus, no packet loss is existing on this protocol. On the other hand, M ATTEMPT to provide no alternative routes for dead nodes. Also, the load of nodes is not uniformed which cause more energy consumptions[15].

RE-ATTEMPT [15] is similar to ATTEMPT with the following enhancements in order to increase the stability of the network, reduce packet loss and reduce energy consumption. First, the protocol supports data priority concept in a similar way provided in ATTEMPT by having two transmission ways according to the data type. Moreover, it allocates a fixed time for emergency data in CFP (Contention Free Period) of MAC (Medium Access Control) superframe structure to prevent its competition with other normal data to ensure no delay for emergency data. CAP (Contention Access Period) is assigned for other normal data to be sent one after another. Second, to save energy RE-ATTEMPT disable the hot spot detection process. The simulation showed that RE_ATEEMPT increase the stability and reduce the energy consumption in comparison with ATTEMPT. However, having direct communication between nodes and sink for urgent data is not the optimal way to reduce the delay as the long-distance will increase the packet loss [2]. This 


\title{
International Journal of Advanced Research in Computer and Communication Engineering
}

\author{
Vol. 8, Issue 9, September 2019
}

also affects energy and throughput. Moreover, disabling the hotspot consideration cause a major drawback on this protocol.

Thermal-Aware Localized QoS Routing (TLQoS) [10] is a cross-layer proactive routing which combines the QoS (Quality of Service) with thermal aware routing. It is a modular approach where each module handles a type of data. TLQoS divides the traffic flow to four types: First, critical traffic which includes the data which needs minimum delay and maximum reliability (no packet loss) such as real-time data. Second, delay constrained traffic which includes the data required minimum delay but tolerate packet loss such as video streaming applications. Third, reliability constrained traffic which includes the data with no packet loss but a delay is acceptable such as monitoring $\mathrm{pH}-\mathrm{level}$. Fourth, regular traffic which accepts delay and packet loss more than the previous types such as monitoring the temperature in regular vital sign monitoring applications. Potential based routing policy in used for this protocol to determine the best route based on hop count potential, delay potential, reliability potential and temperature potential. Each potential is calculated using its related module. Then, the route is selected as per the maximum values of force function calculated of the potential unless there a hotspot. If hotspot found, another route is selected. The hybrid potential calculation is introduced to avoid infinite loop with different scenarios provided on the paper.

The simulation results showed that the protocol has a significant impact on average end-to-end delay, packet delivery ratio, reliability average temperature rise, and average energy consumption in comparison with TARA, ALTR and TMQoS. However, the overhead is too high [10]. Also, it does not handle the mobility which is an important factor in human networks.

Store and carry are a principal for storing the data to be sent in a better environment and it is used in delay tolerant networks (DTNS). Instead of dropping packets that face a hotspot area as in TARA, a mobility-based temperatureaware routing (MTR) [16] is a protocol that uses store and carry concept to store the packet on a mobile node (on arm and legs) when it is expected to reach a hotspot. Then, re-route process is done to submit the packet again to prevent packet loss. In MTR, the next hop selected depends on node temperature, and probability to meet the sink node. MTR consider the temperature and prevent packet loss; however, the hop count metric is not considered in the routing process.

Thermal and energy-aware routing (TEAR) [7]is a protocol aims to avoid increasing node temperature by reducing the number of transmissions. It is a hybrid protocol uses proactive routing in the setup phase and reactive routing in the data transmission phase. The setup phase depends on gradient cost where each node starts with a cost equal to infinity, and sink cost is zero. Starting from the sink, nodes will send hello packet to its neighbors, each neighbor then updates the cost by adding the link quality, its current temperature and power remaining. The transmission power also calculated in this phase. In the routing phase, based on the cost determined on the setup phase the node selects the neighbor which has the minimum cost to forward the packet using the transmission power calculated in the setup phase. After the transmission, the acknowledgment is sent back from the receiver which contains the updated cost, so the process of broadcasting the hello packet will not be repeated unless the topology changed, hence the number of transmissions will be less, so the efficiency of consuming energy and reducing the increase of temperature. The results of simulation compared with one of the transmission power control protocols and show reducing the number of heated nodes to $70 \%$, increase the throughput and low packet loss as the protocol is considering the link quality for routing decision. However, the protocol not considering the hop count in the routing process.

Adaptive thermal aware routing (ATAR) in [12]is another reactive routing uses Multi-Ring Routing approach to adopt with raising in temperature by finding another path. The algorithm set the nodes into levels according to its distance from the sink node. Each node after the setup phase will have the information about its neighbors in terms of route temperature, and its distance from the sink node. In the data transmission phase, the decision for next-hop will depend on the route temperatures with ring level to ensure that the packet is forwarded toward the sink node.

Thermal aware and energy-optimized routing (TAEO-A) in [17] is proposed to reduce the effect of heat and increase reliability. In the routing phase, the node is selected as per there parameters: remaining energy, the current value of temperature, and distance from the sink node. The results show that the protocol increases the lifetime, throughput and network stability. However, data priority is not considered which is important in monitoring application for the medical field.

Table 1 shows a comparison between the listed protocols where a comparison is done between them. 
Vol. 8, Issue 9, September 2019

Table 1: Comparison between Thermal-aware Routing Protocols in WBAN

\begin{tabular}{|c|c|c|c|c|c|c|c|c|}
\hline Protocol & $\begin{array}{l}\text { Routing } \\
\text { Class }\end{array}$ & Phases & $\begin{array}{c}\text { Routing } \\
\text { Decision } \\
\text { Depends on }\end{array}$ & Data Type & $\begin{array}{l}\text { Hotspot } \\
\text { Handin } \\
\quad \mathrm{g}\end{array}$ & $\begin{array}{l}\text { Ther } \\
\text { mal } \\
\text { Metho } \\
\text { d }\end{array}$ & $\begin{array}{l}\text { Measure } \\
\text { Node } \\
\text { Temperatu } \\
\text { re }\end{array}$ & Limitation \\
\hline ATAR [12] & Reactive & $\begin{array}{l}\text { - Initialization phase } \\
\text { - Data transmission }\end{array}$ & $\begin{array}{l}\text { Node } \\
\text { Temperature }\end{array}$ & Not applicable & $\begin{array}{l}\text { Detectio } \\
\mathrm{n}\end{array}$ & SAR & $\begin{array}{l}\text { Data } \\
\text { transmission }\end{array}$ & $\begin{array}{l}\text { - Priority of data is not } \\
\text { handled } \\
\text { - Increased overhead for } \\
\text { ring levels [7] }\end{array}$ \\
\hline TEAR [7] & $\begin{array}{l}\text { Hybrid } \\
\text { Model }\end{array}$ & $\begin{array}{l}\text { - Setup phase } \\
\text { - Data transmission }\end{array}$ & $\begin{array}{l}\text {-Node } \\
\text { temperature } \\
\text {-Link quality } \\
\text {-Residual } \\
\text { energy }\end{array}$ & Not applicable & $\begin{array}{l}\text { Avoidan } \\
\text { ce }\end{array}$ & SAR & $\begin{array}{l}\text { Frequency } \\
\text { of } \\
\text { transmission } \\
\text { and } \\
\text { reception }\end{array}$ & $\begin{array}{l}\text { - Not adapt with topology } \\
\text { change } \\
\text { - Priority of data is not } \\
\text { handled }\end{array}$ \\
\hline MTR [16] & Reactive & $\begin{array}{l}\text { - Routing for static } \\
\text { nodes } \\
\text { - Routing for } \\
\text { dynamic }\end{array}$ & $\begin{array}{l}\text { - Low } \\
\text { temperature } \\
\text { - High } \\
\text { probability to } \\
\text { meet the sink } \\
\text { node }\end{array}$ & Not applicable & $\begin{array}{l}\text { Avoidan } \\
\text { ce }\end{array}$ & NA & NA & $\begin{array}{l}\text { - Same limitation for LTR } \\
\text { in case of static nodes }\end{array}$ \\
\hline TLQoS [10] & Proactive & $\begin{array}{l}\text { - Initialization phase } \\
\text { - Routing process }\end{array}$ & $\begin{array}{l}\text { Potential } \\
\text { based routing } \\
\text { policy }\end{array}$ & $\begin{array}{l}\text { Using queuing } \\
\text { manager }\end{array}$ & $\begin{array}{l}\text { Avoidan } \\
\text { ce }\end{array}$ & NA & NA & $\begin{array}{l}\text { - Overhead is high } \\
\text { - Not handling human } \\
\text { mobility } \\
\text { - Not guarantee the optimal } \\
\text { route }\end{array}$ \\
\hline $\begin{array}{l}\text { ER- } \\
\text { ATTEMPT } \\
{[15]}\end{array}$ & Proactive & $\begin{array}{l}\text { - Initialization phase } \\
\text { - Routing phase } \\
\text { - Scheduling phase } \\
\text { - Data transmission }\end{array}$ & $\begin{array}{l}\text { - Data priority } \\
\text { - Min hop } \\
\text { count to sink }\end{array}$ & $\begin{array}{l}\text { - Normal data } \\
\text { is waiting for } \\
\text { emergency } \\
\text { data } \\
\text { - Direct } \\
\text { communicatio } \\
n \text { if urgent data } \\
\text { - Fixed time } \\
\text { for emergency } \\
\text { data in CFP to } \\
\text { avoid delay }\end{array}$ & $\begin{array}{l}\text { Avoidan } \\
\text { ce }\end{array}$ & SAR & $\begin{array}{l}\text { Hot spot } \\
\text { detection is } \\
\text { disabled }\end{array}$ & $\begin{array}{l}\text { - Direct communication } \\
\text { increases packet loss and } \\
\text { consume energy. }\end{array}$ \\
\hline $\begin{array}{l}\text { M_ATTEM } \\
\text { PT }[14]\end{array}$ & Proactive & $\begin{array}{l}\text { - Initialization phase } \\
\text { - Routing phase } \\
\text { - Scheduling phase } \\
\text { - Data transmission } \\
\text { - Invitation phase for } \\
\text { link breakage }\end{array}$ & $\begin{array}{l}\text { - Min hop } \\
\text { count to sink } \\
\text { - Min energy } \\
\text { route }\end{array}$ & $\begin{array}{l}\text { - Normal data } \\
\text { waiting for } \\
\text { emergency } \\
\text { data } \\
\text { - Direct } \\
\text { communicatio } \\
\mathrm{n} \text { if urgent data }\end{array}$ & $\begin{array}{l}\text { Avoidan } \\
\text { ce }\end{array}$ & SAR & $\begin{array}{l}\text { data } \\
\text { communicat } \\
\text { ion }\end{array}$ & $\begin{array}{l}\text { - No alternative routes for } \\
\text { dead nodes } \\
\text { - Load of nodes is not } \\
\text { uniformed, so more energy } \\
\text { consumption. }\end{array}$ \\
\hline $\begin{array}{c}\text { ATTEMPT } \\
{[14]}\end{array}$ & Proactive & $\begin{array}{l}\text { - initialization phase } \\
\text { - routing phase } \\
\text { - scheduling phase } \\
\text { - data transmission } \\
\text { phase }\end{array}$ & $\begin{array}{l}\text { - min hop } \\
\text { count to sink } \\
\text { - min energy } \\
\text { route }\end{array}$ & $\begin{array}{l}\text { - Normal data } \\
\text { waiting for } \\
\text { emergency } \\
\text { data } \\
\text { - Direct if } \\
\text { urgent data }\end{array}$ & $\begin{array}{l}\text { Avoidan } \\
\text { ce }\end{array}$ & SAR & $\begin{array}{l}\text { data } \\
\text { communicat } \\
\text { ion }\end{array}$ & $\begin{array}{l}\text { - No mobility handling } \\
\text { - No alternative for dead } \\
\text { routes } \\
\text { - Load of nodes is not } \\
\text { uniformed }\end{array}$ \\
\hline LTRT [13] & Proactive & $\begin{array}{l}\text { - Assign temperature } \\
\text { - calculating routes } \\
\text { - Apply single- } \\
\text { source shortest path } \\
\text { algorithms } \\
\text { - Maintenance of } \\
\text { routing periodically }\end{array}$ & $\begin{array}{l}\text { Min } \\
\text { temperature } \\
\text { route by using } \\
\text { graph. }\end{array}$ & Not applicable & $\begin{array}{l}\text { Avoidan } \\
\text { ce }\end{array}$ & NA & NA & $\begin{array}{l}\text { - It increases the overhead } \\
\text { as node needs to know the } \\
\text { temperature of all other } \\
\text { nodes. } \\
\text { - No data priority is handled } \\
\text { - Delay or reliability of the } \\
\text { path is not considered }\end{array}$ \\
\hline LTR [11] & Proactive & $\begin{array}{l}\text { - Setup Phase: } \\
\text { - Routing phase }\end{array}$ & $\begin{array}{l}\text { - Coolest } \\
\text { neighbour } \\
\text { - MAX-Hop } \\
\text { count }\end{array}$ & Not applicable & $\begin{array}{l}\text { Avoidan } \\
\text { ce }\end{array}$ & NA & $\begin{array}{l}\text { data } \\
\text { communicat } \\
\text { ion }\end{array}$ & $\begin{array}{l}\text { - Not considering the full } \\
\text { route temperature. } \\
\text { - High overall network } \\
\text { temperature. - Delay for the } \\
\text { packet delivery } \\
\text { - Increase the hop count }\end{array}$ \\
\hline ALTR [11] & Proactive & $\begin{array}{l}\text { - Setup phase } \\
\text { - Routing phase }\end{array}$ & $\begin{array}{l}\text { Coolest } \\
\text { neighbour } \\
\text { MAX-Hop }\end{array}$ & Not applicable & $\begin{array}{l}\text { Avoidan } \\
\text { ce }\end{array}$ & NA & $\begin{array}{l}\text { data } \\
\text { communicat } \\
\text { ion }\end{array}$ & $\begin{array}{l}\text { - Not considering the full } \\
\text { route temperature } \\
\text { - Waste network bandwidth }\end{array}$ \\
\hline
\end{tabular}


Vol. 8, Issue 9, September 2019

\begin{tabular}{|c|c|c|c|c|c|c|c|c|}
\hline & & & $\begin{array}{l}\text { count } \\
\text { MAX_HOPS_ } \\
\text { ADAPTIVE }\end{array}$ & & & & & $\begin{array}{l}\text { through unnecessary } \\
\text { transmissions } \\
\text { - Packet traverse through } \\
\text { hotspot }\end{array}$ \\
\hline TARA [6] & Proactive & $\begin{array}{l}\text { - Setup phase } \\
\text { - Data forwarding } \\
\text { - Hotspot detection }\end{array}$ & $\begin{array}{l}\text { Temperature } \\
\text { for next hop }\end{array}$ & Not applicable & $\begin{array}{l}\text { Detectio } \\
\mathrm{n}\end{array}$ & FDTD & $\begin{array}{l}\text { number of } \\
\text { packets } \\
\text { transmitted } \\
\text { and received } \\
\text { by the node }\end{array}$ & $\begin{array}{l}\text { - Not considering the full } \\
\text { route temperature } \\
\text { - High end-to-end delay. } \\
\text { - Lower reliability } \\
\text { - High energy consumption } \\
\text { - Packet traverse through } \\
\text { hotspot }\end{array}$ \\
\hline TAEO [17] & Proactive & $\begin{array}{l}\text { - Initialization } \\
\text { - Routing } \\
\text { - Scheduling }\end{array}$ & $\begin{array}{l}\text { - max energy } \\
\text { - min } \\
\text { temperature } \\
\text { - min distance } \\
\text { to sink }\end{array}$ & Not applicable & $\begin{array}{l}\text { Avoidan } \\
\text { ce }\end{array}$ & SAR & $\begin{array}{l}\Delta \mathrm{T}=(\mathrm{SAR}) \mathrm{t} \\
/ \mathrm{C}\end{array}$ & $\begin{array}{l}\text { - Mobility is not considered. } \\
\text {-Not handling data priority }\end{array}$ \\
\hline
\end{tabular}

\section{IV.TOOLS AND APPLICATIONS}

As per the literature, OMNET++ with Castalia simulator, MATLAB, and C++ are the most commonly used tools for simulation for WBAN routing in general and thermal aware routing as an important branch. However, there exists another tool which could be used such as OPNET, C, NetSim and TinyOS.

WBAN is getting more attention in the coming days for developing new applications in the medical field. Different requirements are needed for medical applications. For example, continuous monitoring applications tolerate the packet loss parameter and more likely to be negatively affected by the delay. However, the delay is catastrophic if abnormal behavior is detected and needs to be communicated with the medical center to take the needed action to save the patient. Thus, choosing the protocol for a WBAN system depends on the particular application of the system as stated by [18]. However, the effect of heating over human tissue should be considered in all inter/intrabody applications.

Thermal aware routing is still an open research question for WBAN especially in the medical field in order to find the optimal routing protocol that minimizes heating effects on human tissues, reducing energy consumption, ensure no delay for critical data, handling human mobility, reduce packet loss and increase network lifetime.

\section{Challenges and Future Research}

As previously mentioned, WBAN is handling environments with limited resources in terms of energy, and transmission power. In healthcare applications, the human body is adding more challenges on the routing process due to human limb movements, sensitivity to the heat generated by the WBAN devices. Following are the routing design challenges in routing protocols.

\section{- $\quad$ Biocompatibility}

The sensor devices need to be compatible with human body environments. For example, underwater sensors could be introduced with thermal effects as new research for such a network. Also, the topology changes which is affected that organ is changing in shape due to human different activities.

\section{- Reducing Hop count}

Despite the existence of routing protocols that depend on single-hop communication, multi-hop is more efficient in terms of energy consumption [19]. However, increasing the hop count may cause delay and packet loss. The balance and trade-off between parameters to ensure quick delivery with minimum energy consumption is needed.

- $\quad$ Supporting Data Priority

As stated in [4] that IEEE 805.11.6 is not considering the data priority. The nature of the human body in the medical field in force the need for data priority to handle the critical real-time applications. Such concept in WBAN for the medical application have to ensure a balance between the time, the increase of heating and energy consumption. Direct transmission for emergency data will provide fast transmission; however, it increases energy consumption in comparison with multi-hop communication and vice versa.

- $\quad$ Temperature Awareness to Avoid Tissue Damage

There is a need for temperature consideration for WBAN in medical applications. This includes avoidance techniques such as TEAR which reduce the number of transmissions to avoid increasing the heat for nodes. The other techniques which detect the hotspot node like TARA that withdraw the packet if reached a hotspot node. The calculations for detecting or avoiding the hotspot nodes increase the overhead and complexity of the network. 


\title{
International Journal of Advanced Research in Computer and Communication Engineering
}

\author{
Vol. 8, Issue 9, September 2019
}

\section{- $\quad$ Supporting Mobility}

To adapt to the human body movement, mobility in routing is an important factor that needs to be considered. The change in node location, hence the topology changes which cause link breakages. This needs to be addressed for more reliable network and it will affect the real-time applications where the fast response is a major concern.

- Fault-Tolerant

In order to allow wide use of WBAN in critical medical applications. Different fault tolerance techniques could be added to increase the reliability of the routing protocol.

- Handling Resource limitation

Resources for WBAN networks include energy, commutation tasks and transmission power. Saving the limited resources for WBAN will increase the robustness needed to ensure the longest period of working without replacement.

Therefore, finding the optimal routing protocol that satisfies critical application in medical fields is still and open questions.

\section{CONCLUSION}

Promising revolution in medical health care applications is expected by using WBAN. It is used for healthcare monitoring, early diagnosis and detection for disses in its primary stage, or therapeutics monitoring. The human body is a restrictive environment considered by WBAN different protocols. In this paper, literature of thermal aware routing protocols is provided as different ways for dealing with increasing heat to avoid tissue damage. A comparison between the listed protocols is provided in terms of the type of routing, a method to calculate the rise in temperature, and routing decision parameter and other routing parameters. The limitation for each routing is also provided to help future optimization. Finally, the main parameters which need consideration in WBAN routing in health care are presented and the trade-off between them is shown.

In conclusion, routing is still an open research question for WBAN especially in the medical field in order to find the optimal routing protocol that could provide the lowest delay, reduce packet loss, handling the effect of temperature to avoid damage human tissues, adaptive to human movement. It also should be fault tolerance to support the usage in critical applications.

\section{REFERENCES}

[1]. R. Negra, I. Jemili, and A. Belghith, "Wireless body area networks: Applications and technologies," Procedia Computer Science, vol. 83, pp. 1274-1281, 2016.

[2]. Y. Qu, G. Zheng, H. Ma, X. Wang, B. Ji, and H. Wu, “A survey of routing protocols in WBAN for healthcare applications," Sensors, vol. 19, no. 7 , pp. 1638,2019 .

[3]. C. Chakraborty, B. Gupta, and S. K. Ghosh, "A review on telemedicine-based WBAN framework for patient monitoring," Telemedicine journal and e-health : the official journal of the American Telemedicine Association, vol. 19, no. 8, pp. 619-626, 2013.

[4]. S. Rashwand, J. Mišić, and H. Khazaei, "IEEE 802.15. 6 under saturation: Some problems to be expected," Journal of Communications and Networks, vol. 13, no. 2, pp. 142-148, 2011

[5]. B. Romaissa, and B. D. Eddine, "In-Body Routing Protocols for Wireless Body Sensor Networks." pp. 160-165.

[6]. Q. Tang, N. Tummala, S. K. Gupta, and L. Schwiebert, "TARA: thermal-aware routing algorithm for implanted sensor networks." pp. $206-217$.

[7]. G. Ahmed, D. Mahmood, and S. Islam, "Thermal and energy aware routing in wireless body area networks," International Journal of Distributed Sensor Networks, vol. 15, no. 6, pp. 1550147719854974, 2019.

[8]. D. Sullivan, Electromagnetic Simulation Using the FDTD Method, 2nd Edition: Wiley-IEEE Press, 2000.

[9]. M. M. Monowar, and F. Bajaber, "Towards Differentiated Rate Control for Congestion and Hotspot Avoidance in Implantable Wireless Body Area Networks,” IEEE Access, vol. 5, pp. 10209-10221, 2017.

[10]. M. M. Monowar, and F. Bajaber, "On Designing Thermal-Aware Localized QoS Routing Protocol for in-vivo Sensor Nodes in Wireless Body Area Networks," Sensors (Basel, Switzerland), vol. 15, no. 6, pp. 14016-14044, 2015.

[11]. A. Bag, and M. A. Bassiouni, "Energy efficient thermal aware routing algorithms for embedded biomedical sensor networks." pp. 604-609.

[12]. F. Jamil, M. Iqbal, R. Amin, and D. Kim, “Adaptive Thermal-Aware Routing Protocol for Wireless Body Area Network,” Electronics, vol. 8, no. 1 , pp. 47, 2019.

[13]. D. Takahashi, Y. Xiao, and F. Hu, "LTRT: Least total-route temperature routing for embedded biomedical sensor networks." pp. 641-645.

[14]. N. Javaid, Z. Abbas, M. Fareed, Z. A. Khan, and N. Alrajeh, "M-ATTEMPT: A new energy-efficient routing protocol for wireless body area sensor networks," Procedia Computer Science, vol. 19, pp. 224-231, 2013.

[15]. A. Ahmad, N. Javaid, U. Qasim, M. Ishfaq, Z. A. Khan, and T. A. Alghamdi, "RE-ATTEMPT: a new energy-efficient routing protocol for wireless body area sensor networks," International Journal of Distributed Sensor Networks, vol. 10, no. 4, pp. 464010, 2014

[16]. B.-S. Kim, S. Kang, J. Lim, K. H. Kim, and K.-I. Kim, "A mobility-based temperature-aware routing protocol for wireless body sensor networks." pp. 63-66.

[17]. M. Javed, G. Ahmed, D. Mahmood, M. Raza, K. Ali, and M. Ur-Rehman, "TAEO-A thermal aware \& energy optimized routing protocol for wireless body area networks," Sensors, vol. 19, no. 15, pp. 3275, 2019.

[18]. V. Bhanumathi, and C. Sangeetha, "A guide for the selection of routing protocols in WBAN for healthcare applications," Human-centric Computing and Information Sciences, vol. 7, no. 1, pp. 24, 2017.

[19]. S. K. S. Gupta, S. Lalwani, Y. Prakash, E. Elsharawy, and L. Schwiebert, "Towards a propagation model for wireless biomedical applications." pp. 1993-1997. 


\section{BIOGRAPHY}

Miss Hagar Mahmoud Department of Computer Science, King Abdulaziz University, Jeddah, Saudi Arabia. Received her Bachelor's degree in Computer Science at King Abdul Aziz University with Senior Project title Green Books Netwrok in 2015. She was awarded the Master degree in computer science at King Abdulaziz University (KAU), Currently she is a master student, and her research intrest is wireless network.

Dr. Etimad Fadel Department of Computer Science, King Abdulaziz University, Jeddah, Saudi Arabia. Received her Bachelor's degree in Computer Science at King Abdul Aziz University with Senior Project title ATARES: Arabic Character Analysis and Recognition in 1994. She was awarded the M.Phil.,/Ph.D., degree in computer science at De Montfort University (DMU), Liecester, UK. Currently she is working as an Associate Professor at the Computer Science Department at KAU. This was between 2008 -2019. Her main research interests are Distributed Systems, which are developed based on middleware technology. Currently she is looking into and working on Wireless Networks, Internet of Things and Internet of Nano-Things. In addition, she is working on Smart Grids and HetNets. 